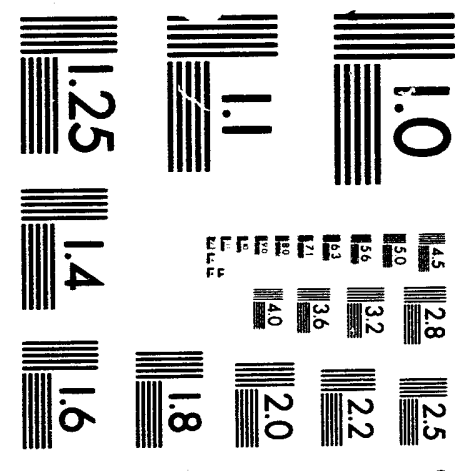



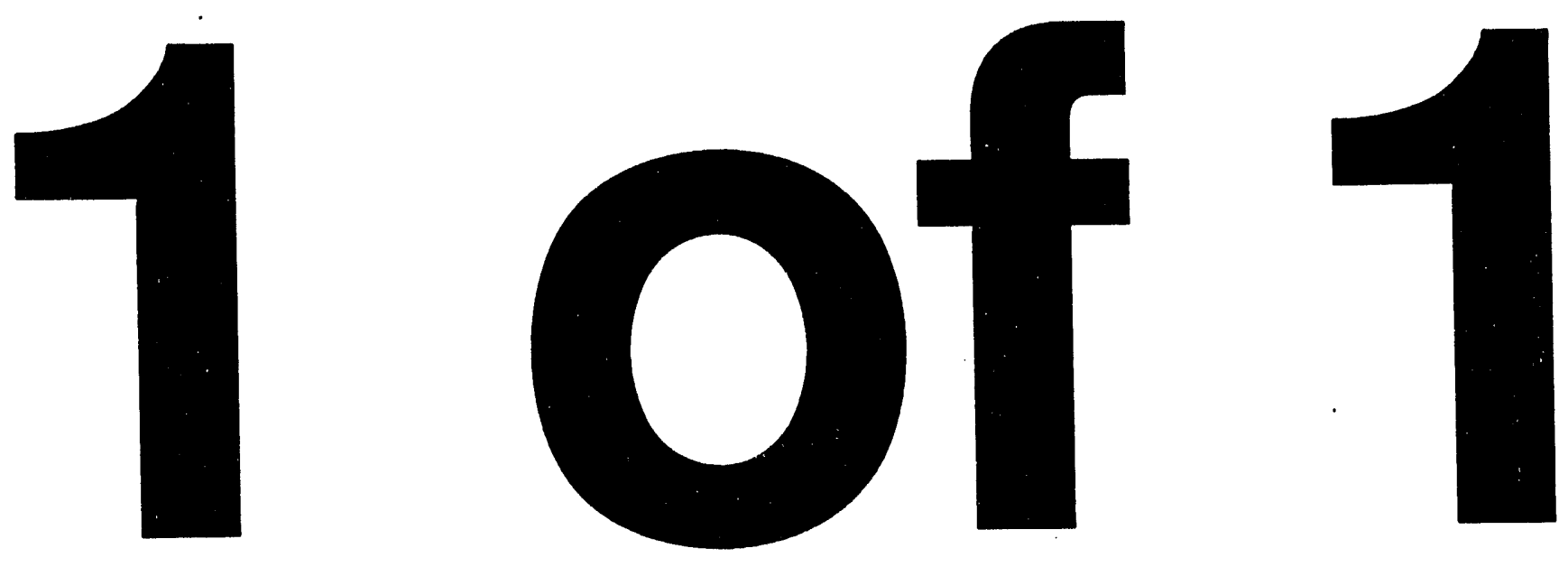
LBL-33798

UC-415

HIFAN-577

The Induction Approach To Heavy Ion Inertial Fusion: Accelerator and Target Considerations

\author{
Roger O. Bangerter \\ Lawrence Berkeley Laboratory \\ Berkeley, CA 94720
}

Submitted to

The International Symposium on Heavy Ion Fusion

Frascati, Italy,

May 25-28 1993

* This work was supported by the Director, Office of Energy Research, Office of Fusion Energy, U.S. Department of Energy under Contract No. DE-AC03-76SF00098. 


\title{
The Induction Approach to Heavy Ion Inertial Fusion: Accelerator and Target Considerations
}

\author{
Roger O. Bangerter \\ Lawrence Berkeley Laboratory \\ 1 Cyclotron Road, MS 47-112 \\ Berkeley, California 94720 USA
}

\begin{abstract}
Induction acceleration is one of two principal approaches for producing ion beams for heavy-ion inertial fusion. This approach was first suggested by the late Denis Keefe of Lawrence Berkeley Laboratory and is the main approach of the U.S. heavy-ion-fusion program. Induction accelerators have the ability to handle high beam currents; therefore, accumulation rings or storage rings are not required. This paper reviews the target and accelerator considerations that are important for the design of induction accelerators for fusion. These considerations, including some important assumptions, have led to a standard induction accelerator concept; however, a careful examination of the assumptions and considerations shows that many of them are not truly fundamental. Through improvements in technology, changes in design, and alternate ways of focusing beams, it appears possible to circumvent or relax the constraints imposed by the standard orthodoxy. If it is possible, it will lead to induction accelerators that are more efficient and less costly than the standard concept.
\end{abstract}

PACS \#41.75Ak

\section{INTRODUCTION}

Induction acceleration, one of the two principal approaches to heavy ion fusion, differs from the other approach, r.f. acceleration, in several important ways. Induction accelerators are generally high-current, low-impedance machines whereas r.f. accelerators are low-current, highimpedance machines. Several factors contribute to this difference. In an ion induction accelerator, one usually accelerates a single, relatively long ion bunch. The charge per unit length, $\lambda$, is

* This work was supported by the Director, Office of Energy Research, Office of Fusion Energy, U.S. Department of Energy under Contract No. DE-AC03-76SF00098. 
approximately constant over the length of the bunch. The current is the product of $\lambda$ and the beam velocity, $v$, so the current increases naturally as the beam velocity increases, even if the bunch maintains a constant length. In fact, if the beam is held together transversely against its space charge forces by a magnetic focusing system, it is possible to increase $\lambda$ because the focusing force increases with increasing velocity. The increase in $\lambda$ is accomplished by slightly ramping the acceleration voltage to compress the beam axially. Because of these effects the current in an ion induction accelerator can increase by large factors as the beam is accelerated. In contrast, the average current in an r.f. linac is constant throughout the length of the accelerator. It is the product of charge per r.f. bucket and the frequency. Since both are fixed, the current is constant. The only practical way to increase the current is to funnel beams. For example, two linacs operating at 160 $\mathrm{MHz}$ could be used to fill alternate buckets of a linac operating at $320 \mathrm{MHz}$.

A second factor leading to the difference in current is the bunch structure. In an r.f. linac each bunch occupies only a fraction of an r.f. cycle leading to a lower average current than could be transported in a continuous pulse. Finally, the cost of r.f. power is an important constraint. For example, accelerating even 1 ampere of current to a kinetic energy of $10 \mathrm{GeV}$ requires a power of $10^{10}$ watts. The cost is prohibitive. The only economical way to operate an r.f. accelerator for heavy ion fusion (HIF) is to accelerate long, low-current pulse trains. The pulsed power used in induction accelerators is much less expensive.

Because of the low current carrying capability of r.f. linacs, it is necessary to multiply the current by multi-turn injection into a series of accumulator or storage rings to get the power required to drive a target. Induction accelerators can carry enough current that storage rings are not needed. Thus induction accelerators appear to have the advantage of simplicity; however, there is far more practical experience with r.f. accelerators. For the power levels needed for fusion there is no experience with either type of accelerator. It is certainly too early to choose between the approaches. 


\section{RELATIONSHIPS AMONG TARGETS, REACTORS, AND ACCELERATORS}

Target, reactor, and accelerator studies in the U.S. have led to a "standard" induction accelerator concept shown in Figure 1. There are many variations of this concept, but most of them have of the order of 10 beams in the magnetic focusing section and produce ion beams with a kinetic energy of the order of $10 \mathrm{GeV}$. The total beam energy is typically several megajoules, and the focal spot radius is 2 or $3 \mathrm{~mm}$. Some of the designs omit beam combining and a few use only electric or magnetic focusing rather than both. Nevertheless most of the designs are basically very similar. This paper will review the physical considerations and assumption that lead to this standard design. There is considerable room for improvement in physics and technology. These improvements can strongly affect the present assumptions. It is therefore likely that the standard concept is not optimal.

Accelerator design begins with target requirements. One is immediately faced with a choice between tirectly and indirectly driven targets. The requirements are substantially different for the two cases. Programatically indirect drive has an advantage because the implosion physics is common to all types of drivers. The information obtained from the laser and light ion programs is directly applicable to HIF. Also, at this time, most of the target physicists in the U.S. believe that indirect drive is more conservative.

Even if one chooses indirect drive, one is still faced with the choice of illumination geometry. In the U.S. we have often adopted two-sided illumination as the standard case. Twosided illumination is compatible with most reactor designs and it is believed to be more conservative than one-sided illumination.

During recent years the issue of illumination geometry has created considerable confusion and controversy. Not everyone believes that one- or two-sided illumination is possible or desirable. There is also discussion of the relative advantages and disadvantages of "filamentary" versus "diffuse" radiators. Some have suggested that two-sided illumination requires filamentary radiatiors. This suggestion is not correct.. Livermore scientists have consistently published gain curves for two-sided targets that accept focal spot radii as large as $5 \mathrm{~mm}$. An object $1 \mathrm{~cm}$ in 
diameter can hardly be described as filamentary. Since the issue of illumination geometry has not been resolved to everyone's satisfaction, it may be useful to digress from the main topic of this paper, induction accelerators, to pose the target problem in a general way.

From a target standpoint, specific energy deposition (energy per unit mass) is a quantity of fundamental importance. Unless a certain specific energy is achieved, it is impossible or inefficient to attain the needed implosion velocity. The energy must be delivered in a short time, so some authors have used specific power rather than specific energy as a figure of merit. The use of specific energy has some advantages because the required value of specific energy depends only weakly on target size (target input energy). If one scales the size of an arbitrary target by some factor $s$, the mass scales as $\mathrm{s}^{3}$, and the input energy also scales roughly as $\mathrm{s}^{3}$, leaving the specific energy nearly unchanged. The implosion time scales as $\mathbf{s}$ so the specific power scales as $\mathbf{s}^{-1}$. In fact it scales somewhat faster than $\mathrm{s}^{-1}$ because smaller targets require higher implosion velocities.

Consider two general classes of targets, spherical targets that are irradiated relatively uniformly by a large number of beams and axial targets that are irradiated from one or two sides. See Figures 2 and 3. In each case there is some region of radius a (e.g., the fuel) that must not be penetrated by the ion beam. The radius of $a$ beam is $b$ and the outer radius of the target is $c$. Between the inner region and the surface of the target there is some mass distribution $\rho$ that depends only on the spherical radius for spherical targets, but may depend on $\mathrm{r}$ and $\mathrm{z}$ (in polar coordinates) for axial targets. If $\rho(r)$ is chosen such that the ions stop in the target, the mass $M$ heated by the beams in the spherical case satisfies $4 \pi a^{2} R \leq M \leq 4 \pi c^{2} R$ where $R$ is the ion range. The lower limit occurs when all the mass is at $r=a$, and the upper limit occurs when all the mass is at $r=c$. For these targets $M$ is independent of $b$, in an average sense, as long as $b<c$.

In the two-sided case, we obtain $M \geq 2 \pi a^{2} R+\pi\left(b^{2}-a^{2}\right) R=\pi\left(a^{2}+b^{2}\right) R$ for $b \geq a$ and $M=2 \pi b^{2} R$ for $b \leq a$.

For one-sided targets we obtain $M=\pi b^{2} R$.

In the axial cases $M$ depends strongly on beam size and $M$ is usually much smaller than in the spherical case. Therefore, for a given beam size, the axial targets have a large advantage in 
terms of specific energy. One can easily show that the axial targets also have a large advantage in intensity (power per unit area), another critically important quantity. However one must usually pay an energy penalty to achieve adequate implosion symmetry if the target is not uniformly irradiated. The fundamental question is:

Does the energy penalty associated with lack of spherical illumination more than offset the axial systems' advantages in specific energy and intensity?

The answer to this question has far-reaching implications for reactor design, emittance constraints, choice of ion kinetic energy, and the desirability of non-Liouvillian manipulations. In any case, most accelerator designs in the U.S. have assumed two-sided illumination.

Even if one chooses two-sided, indirectly driven targets, there is flexibility in the choice of ion range and focal spot size. The flexibility is constrained by the requirement on specific energy, but one can choose large range and a small focal spot or vice versa or anything between. The choice is an accelerator and focusing issue rather than a target issue. The "standard" choice of 10 $\mathrm{GeV}$ heavy ions and a 2 to $3 \mathrm{~mm}$ focal spot radius is not determined by target physics. From a target standpoint ion ranges between about 0.02 and $0.2 \mathrm{~g} / \mathrm{cm}^{2}$ are acceptable.

\section{ACCELERATOR CONSIDERATIONS}

The standard induction accelerator concept has evolved from a number of studies that optimize the accelerator, target, and reactor to obtain the minimum cost of electricity. The following ten considerations in various forms are often assumed to be the principal considerations for the accelerator itself:

1. Induction core relationships.

2. Limits on acceleration gradient.

3. The Maschke limit on transportable current. 
4. The properties of magnetic quadrupoles.

5. The properties of electrostatic quadrupoles.

6. Limits on the number of beams imposed by complexity.

7. The space-charge limit at final focus.

8. Limits on transverse and longitudinal emittance at final focus.

9. Limits on current density and emittance at the ion source.

10. Longitudinal stability.

Many of these considerations are coupled to each other. For example, space charge forces and transverse and longitudinal emittance jointly determine the minimum attainable focal spot size. We will now examine each of these considerations in turn. The purpose of the examination is not to provide rigorous, detailed information on induction accelerator design, but to elucidate as simply as possible, the basic ideas. We will show that, although the ten considerations are commonly used in accelerator design, there are important unceriainties in the physics, and many of the considerations are not really fundamental.

\subsection{Induction core relationships}

Figure 4 is a simplified drawing of a toroidal induction core. The pulsing circuit is schematically denoted by a capacitor and a switch, although in practice it is much more complicated. The ferromagnetic toroid has length $\ell$, inside radius $h$, and outside radius $h+d$. If the core material has a cost per unit volume $\mathrm{c}_{\mathrm{f}}$, the cost of the core is given by $C=c_{f} \pi \ell\left[(h+d)^{2}-h^{2}\right]=c_{f} \pi \ell d(2 h+d)$.

The pulsers produce a flux swing $\Delta \mathrm{B}$ in the ferromagnetic material. In this process some energy will be lost because of hysteresis and eddy currents. The loss depends on $\Delta \mathrm{B}$ and pulse length $\tau$. Let $w(\Delta B, \tau)$ be the energy loss per unit volume. The total energy loss is $\mathrm{E}_{\mathrm{L}}=\mathrm{w}(\Delta \mathrm{B}, \tau) \pi \ell \mathrm{d}(2 \mathrm{~h}+\mathrm{d})$. Faraday's law gives the acceleration voltage produced by the core, $\mathrm{V}_{\mathrm{c}}=\ell \mathrm{d} \mathrm{dB} / \mathrm{dt}$. For induction accelerators $\mathrm{V}_{\mathrm{c}}$ is approximately constant during the pulse so that 
$V_{c}=\ell d \Delta B / \tau$. The energy gained by the beam (or beams) passing through the core is $E_{g}=I V_{c} \tau=I \ell d \Delta B$ where $I$ is the total beam current. The efficiency of the core is $\eta=\frac{E_{g}}{E_{g}+E_{L}}=\frac{I \Delta B}{I \Delta B+w \pi(2 h+d)}$. This simple analysis has ignored several factors such as the finite rise and fall times of the pulse. Nevertheless it does give some important insight. The quantities $\mathrm{c}_{\mathrm{f}}, \mathrm{w}$, and $\Delta \mathrm{B}$ are fixed by the cost and properties of the material. To minimize cost and maximize efficiency, one would like to minimize $h$ and maximize $I$. Of course any optimization must be done with global constraints in mind. It does no good to maximize current in the accelerator if the current becomes too high to focus. Limits on current in the accelerator and focusing system will be discussed below.

Since the energy gain is proportional to $\ell \mathrm{d}$, while the cost and energy loss are proportional to $\ell d(2 h+d)$, it might appear desirable to make $d$ small and $\ell$ large. There is a limit to this strategy. The beam must be held together transversely by a focusing system. Large $\ell$ leads to a long accelerator and high cost for the beam focusing system. There is clearly some economic optimum. Unfortunately the economic optimum may lie outside a limit on acceleration gradient as will now be explained.

\subsection{Limits on acceleration gradient.}

As one increases $d$ with $\ell, \Delta B$, and $\tau$ fixed, the voltage $V_{c}$ and the acceleration gradient increase. At some point the gradient exceeds the breakdown limit. There is little experience with high-voltage, long-pulse ion induction linacs; therefore, the breakdown limit is uncertain. The HIF studies based on induction linacs have used rules-of-thumb such as $E<(1 \mu \mathrm{s} / \tau)^{\alpha} \mathrm{MeV} / \mathrm{m}$ where $\alpha$ is in the range of 0.5 to 1 . This constraint is limiting over a significant fraction of the accelerator. While such rules-of-thumb are reasonable, based on experience with short-pulse electron induction linacs, more precise limits must be measured. 
3.3 The Maschke current limit.

This limit is based on the simple fact that the space-charge force of a beam cannot exceed the applied focusing force. Maschke originally conservatively assumed that it could not exceed one half the applied force, but experience and simulations have shown that it can nearly equal the applied force. The Maschke limit has been written in a variety of forms and has led to considerable confusion because different authors hold different things constant. We will give a brief description of the physical considerations that are important.

The envelope equation can be written (nonrelativistically) as

$$
\frac{d^{2} a}{d z^{2}}=-k^{2} a+\frac{\varepsilon^{2}}{a^{3}}+\frac{\text { Iqe }}{2 \pi \varepsilon_{0} m v^{3} a}
$$

where $\mathrm{a}$ is beam size, $\mathrm{z}$ is the coordinate along the beam axis, $\mathrm{k}$ is the wavenumber produced by the focusing fields, $\varepsilon$ is unnormalized emittance, $m$ is mass, and $q$ is charge state. For simplicity we consider a smooth approximation where $\mathrm{k}_{0}$ is the wavenumber of the quasi-harmonic oscillations in the quadrupole focusing channel. Specifically we set $k_{0}=\frac{\sigma_{0}}{2 \mathrm{~L}}$ where $\sigma_{0}$ is the phase advance in a period of length $2 \mathrm{~L}$ in the absence of space charge. For typical HIF beams the emittance term is small except at final focus. For simplicity we ignore it. By setting $d^{2} a / d z^{2}=0$, corresponding to a beam of constant size, we obtain the current limi $\hat{\imath}$

$$
\mathrm{I}_{\max }=\pi \varepsilon_{0}\left(\frac{\mathrm{mv}^{3}}{2 \mathrm{qe}}\right)\left(\frac{\mathrm{a}}{\mathrm{L}}\right)^{2} \sigma_{0}^{2}
$$

A detailed calculation that does not use the smooth approximation gives a slightly different result, but this form is adequate for our purposes. In this form it appears that the transportable current is proportional to the square of the beam size. There are, however, auxiliary constraints that must be applied to this equation. The maximum current occurs when the beam fill the channel, but simulations show that image forces cause unacceptable emittance growth if the beam radius $a$ is 
more than about $80 \%$ of the aperture ap. For magnetic quadrupoles, the phase advance $\sigma_{0}$ is approximately proportional to $\frac{B_{p} \eta L^{2}}{m v a_{p}}$ where $B_{p}$ is the magnetic field at $a_{p}$ and $\eta$ is the occupancy factor, the fraction of the half-period length $L$ occupied by a quadrupole. For electrostatic quadrupoles $B_{p}$ is replaced by $E_{p} / v$ where $E_{p}$ is the electric field at $a_{p}$. For high velocity ions, magnetic focusing is stronger than electrostatic focusing, but at low velocity the $\mathrm{E}_{\mathrm{p}} / \mathrm{v}$ dependence makes electrostatic focusing more effective. This dependence is the icason that the standard concept uses electrostatic focusing at low energy and magnetic focusing at higher energy.

There are other important characteristics of the current limit. If $\sigma_{0}$ is greater than about $\pi / 2$, destructive instabilities arise. If the aspect ratio $a_{p} / L$ is too large, quadrupole focusing doesn't make sense. There are large aberrations in the fringe fields of the quadrupoles [1], and the fields of adjacent quadrupoles tend to cancel.

Under some conditions, usually at the low-energy end of the accelerator, the maximum achievable field becomes the limiting factor. In this case, for fixed aperture, the current is maximized by increasing $L$ to obtain $\sigma_{0}=\pi / 2$. The current limit is then proportional to $B_{\mathrm{p}} \mathrm{a}^{2} / \mathrm{a}_{\mathrm{p}}$ or, since $\mathrm{a}=\mathbf{0 . 8} \mathrm{a}_{\mathrm{p}}$ for maximum current, $\mathrm{I}$ is linearly proportional to $\mathrm{B}_{\mathrm{p}} \mathrm{a}$ or $\mathrm{B}_{\mathrm{p}} \mathrm{a}_{\mathrm{p}}$. Numerically, $I_{\max }=4 \times 10^{5} \mathrm{Ba} \beta^{2}$ amperes (where $\beta=v / c$ ) is a good rule-of-thumb. This scaling has remarkable consequences. Suppose for a moment that the overall transverse dimensions of a quadrupole are proportional to the aperture. The number of beams that can be placed in an induction core having inside radius $h$ goes as $\left(h / a_{p}\right)^{2}$ but the current per beam goes as $a_{p}$. Therefore one maximizes the current in a given core by using a large number of small beams. In fact the equations say that in the limit of very small beams we can transport as much current as we desire in an arbitrarily small aperture $h$, leading to low cost and high efficiency. This scaling was one of the main arguments for developing multi-beam induction linacs for fusion applications. It is clear that $a_{p}=0$ doesn't make sense so we examine other factors that might place a lower limit on beam size. 


\subsection{Limits on magnetic quadrupoles.}

Figure 5 is a simplified diagram of a magnetic quadrupole with 4 windings carrying a current density J. For superconducting materials there is some maximum current density $\mathrm{J}_{\max }$ and some maximum area that can be effectively and economically filled with conductors. This area will scale as $\mathrm{a}^{2}$. On the other hand Ampere's Law, $\int \mathrm{H} \cdot \mathrm{d} \ell=\mathrm{I}$, tells us that the field will scale as $\mathrm{I} / \mathrm{a}$. Therefore in the limit where all available area has been filled by superconductor, the product $B_{p} a$ in the current limit scales as $\mathrm{a}^{2}$, and there is no advantage to very small beams. Moreover there is some thickness of thermal insulation that is needed. Induction accelerators have large sections of room-temperature acceleration gaps so warm-bore magnets must be used. The thickness of thermal insulation is not linearly proportional to beam size, so superconducting magnetic quadrupoles don't scale well to small size for this reason also. There is also an upper limit on beam size. The product $B_{p} a$ will only scale as $\mathrm{a}^{2}$ until $B_{p}$ reaches the limit set by either the superconductor or mechanical strength. All things considered we now believe that an aperture radius of about $6 \mathrm{~cm}$ is optimal for magnetic quadrupole systems. Advances in superconductors or insulation would push this optimum size to smaller values.

\subsection{Limits on electrostatic quadrupoles.}

In multibeam electrostatic quadrupole arrays with cylindrical electrodes, the ratio of the aperture and the electrode radius is usually fixed at 7/8 to minimize the dodecapole component of the electric field. Therefore the assumption that the overall quadrupole dimensions scale with aperture size is correct. Furthermore nearly all experience with high voltage electrostatic devices shows that the attainable field increases with decreasing size, exactly the opposite behavior of small magnetic quadrupoles. In the electrostatic case the minimum size is set by alignment and fabrication tolerances. In a simple model the quadrupole aperture must be increased by some clearance $\delta$ to allow for beam misalignment. In this case, one can analytically derive the optimal quadrupole aperture. The maximum current per beam is proportional to $\mathrm{E}_{\mathrm{p}} \mathrm{a}^{2} / \mathrm{a}$. The area occupied by the beam and the lens is proportional to $a_{p}{ }^{2}$. Assume a power law behavior for $E_{p}$ 
such that $\mathrm{E}_{\mathrm{p}} \propto \mathrm{a}_{\mathrm{p}}^{-\alpha}$ where $\alpha$ is usually in the range of 0 to 0.5 . The current density averaged over the quadrupole is given by $J_{a v e}=g\left(a_{p}-\delta\right)^{2} / a_{p}{ }^{3+\alpha}$ where $g$ is some constant. The value of $a_{p}$ that maximizes $\mathrm{J}_{\text {ave }}$ is given by $\mathrm{a}_{\mathrm{p}}=(3+\alpha) \delta /(1+\alpha)$. For present alignment and fabrication tolerances, the beam might wander as much as $1 \mathrm{~cm}$ off axis unless accurate sensing and steering systems are developed. If $\delta=1 \mathrm{~cm}$, the optimal value for $a_{p}$ is 2.3 to $3 \mathrm{~cm}$. As is the case for magnetic quadrupoles, improvements in technology will push the optimum toward smaller quadrupoles.

Since the optimal size of magnetic quadrupoles is larger than the optimal size of electrostatic quadrupoles, it makes sense to combine beams transversely at the transitions between electrostatic and magnetic focusing as shown in Figure 1. 
3.6 Complexity associated with a large number of beams.

Some accelerator designers arbitrarily place an upper limit on the number of beams. Since there has been excellent progress in automated control in recent years, there is little basis for such a constraint. There are limits on the number of beams, but these limits should be set by economic, engineering, and physics constraints, not prejudice.

\subsection{The space-charge focusing limit.}

For simplicity accelerator designers have often assumed that the envelope equation correctly describes the behavior of beams between the final focusing lenses and the target. If we temporarily ignore emittance, we can readily understand the scaling that comes from such an assumption. In this case, the solution of the envelope equation arises naturally from energy considerations. The change in potential at the edge of a cylindrical beam as the beam is compressed from a radius a to a smaller radius $b$ is given (nonrelativistically) by $\frac{\lambda(1-f) q e}{2 \pi \varepsilon_{0}} \ln \left(\frac{a}{b}\right)$. where $f$ is the neutralization fraction and $\mathrm{q}$ is the charge state.

In the beam rest frame the lenses impart a radially inward velocity that must overcome the potential change. For small convergence angles, the kinetic energy corresponding to this velocity is simply $\mathrm{T} \theta^{2}$ where $\theta$ is the convergence angle from the lens and $\mathrm{T}$ is the total ion kinetic energy. For simple lens systems, $\theta$ must be less than about $15 \mathrm{mr}$ to avoid aberrations. If we set $\theta$ to this maximum value, the maximum power that can be focused, $P=\lambda v T / q$, is proportional to $T^{5 / 2}$. Thus this constraint has a very strong dependence on ion kinetic energy. HIF accelerator designers often conservatively assume that $f=0$. In this case this focusing constraint is usually the most stringent constraint determining ion kinetic energy and the number of beams. In contrast light-ion accelerator designers always assume that $\mathrm{f} \approx 1$. Although the envelope equation is often used, the physics is not correct in the reactor chamber. The charge state of the beam is time dependent because of photoionization and stripping. Moreover the effects of multiple beams are often ignored. We are thus in a curious position. One of the most important constraints for HIF 
accelerators (both r.f. and induction) is neither correct nor fundamental. In fact it is almost completely ignored in light ion fusion. Evidently methods to neutralize the beams in the reactor chamber could have a very large influence on accelerator design.

Calculation of the neutralization and the behavior of the beams in the reactor have been presented at this symposium [2]. This is an important topic that requires additional attention.

\subsection{Limits on transverse and longitudinal emittance.}

The beams must be focused against not only the space-charge term in the envelope equation, but also the emittance term. If the beam convergence angle is limited to $15 \mathrm{mr}$ as described above, the emittance must satisfy $\varepsilon \leq 45 \pi \mathrm{mm} \cdot \mathrm{mr}$ to give a $3 \mathrm{~mm}$ focal spot radius. For an ion beam with $\beta=1 / 3$, this corresponds to a normalized emittance $\varepsilon_{N} \leq 15 \pi \mathrm{mm} \cdot \mathrm{mr}$. If one also includes the space charge forces, $\varepsilon_{\mathrm{N}} \leq 10 \pi \mathrm{mm} \cdot \mathrm{mr}$ is a more reasonable limit. On the other hand the limit $\theta \leq 15 \mathrm{mr}$ is based on simple lens systems that employ only quadrupoles and dipoles. D. Ho and coworkers have designed a focusing system that uses octupoles to correct aberrations [3]. AccSys Technologies has fabricated the lenses for such a system and experimental results should be available soon.

There is also a limit on longitudinal emittance. Typical uncorrected lens systems have excessive chromatic aberrations if the momentum spread $\delta p / p$ exceeds about $0.5 \%$. If $\delta p / p=$ $0.5 \%$, the energy spread $\delta \mathrm{T}$ is $1 \%$. Since the final pulse duration $\tau$ is about $10 \mathrm{~ns}$, the longitudinal emittance limit for a $10 \mathrm{GeV}$ beam is $\varepsilon_{\mathrm{L}}=\delta \mathrm{T} \tau=(1 \%)(10 \mathrm{GeV})(10 \mathrm{~ns})=1 \mathrm{eV} \cdot \mathrm{s}$. Chromatic aberrations can, in principle, be corrected; therefore, this limit is not truly fundamental. However no one has yet designed a practical achromatic system for HIF.

The transverse emittance limit has an interesting property. It is nearly independent of ion kinetic energy. The specific energy in the target depends on $b^{2} R$. For $5-10 \mathrm{GeV}$ heavy ions $R$ is approximately proportional to $T$. Therefore we have $b^{2} R \propto b^{2} T \propto \frac{\varepsilon_{N}^{2} T}{\beta^{2} \theta^{2}} \propto \frac{\varepsilon_{N}{ }^{2}}{\theta^{2}}$, so for fixed $\theta$ the specific energy is independent of $T$. The decrease in range at lower kinetic energy just 
compensates for the increase in focal spot radius. Similar arguments show that the constraint on longitudinal emittance becomes more stringent with decreasing kinetic energy.

3.9 Limits on current density and emittance at the ion source.

Sources that satisfy these limits are presently available; however, they are restricted to a few special ions. Additional source development would allow a wider choice of ions.

\subsection{Longitudinal stability.}

Many induction linac designs have ignored this issue. It is assumed that longitudinal instability can be controlled by building pulsers that correct any instability growth. [4].

The considerations and constraints explained above can be grouped into three categories. The first four, the induction core relationships, the acceleration gradient, the current limit, and the properties of magnetic quadrupoles are relatively fundamental. Improvements in ferromagnetic materials, superconductors, and thermal and electric insulators can be expected, but it is difficult to circumvent or repeal the basic physics.

The next four, the properties of electrostatic quadrupoles, complexity, the space-charge focusing limit, and emittance, are less fundamental. They can be relaxed or circumvented by more accurate fabrication, design improvements, or alternate ways of doing things, for example, corrected lens systems or neutralization. It is noteworthy that the focusing constraint, perhaps the most stringent constraint for heavy ion fusion, is a constraint that is not usually assumed to be limiting in light ion fusion despite the fact that neutralization must be much more complete in the light ion case.

The final category, ion sources and longitudinal instability are not considered limiting, but more development would be useful.

It is interesting that improvements and progress in nearly all ten areas will allow accelerator designs to move to higher current and efficiency and lower kinetic energy and cost, but ultimately 
the greatest reduction in the cost of an induction accelerator may require a new accelerator configuration such as recirculation [5].

\section{PLANS AND CONCLUSIONS}

In order to resolve the physics and technology issues and decide among the various accelerator configurations, an experimental program is needed. Lawrence Berkeley Laboratory, Lawrence Livermore National Laboratory, and industrial partners have proposed building an accelerator known as ILSE (Induction Linac Systems Experiments) to address nearly all the topics discussed in this paper, including recirculation. The ILSE beams will be driver scale in terms of $a_{p}$ and $\lambda$. To save cost, the ILSE kinetic energy (about $10 \mathrm{MeV}$ ) will be lower than the kinetic energy of a driver, and ILSE will have fewer beams. Other details of ILSE are given in these proceedings $[6,7,8,9]$ and have also been published elsewhere [10]. The ILSE Program together with the ongoing r.f. programs, will provide a sound basis for deciding between r.f. and induction acceleration.

In conclusion, nearly two decades of research on targets, reactors, and accelerators has led to a "standard" induction accelerator. Nevertheless many of the assumptions that provide the basis for the standard design remain untested and may be incorrect. It seems likely that there is still considerable room for progress and innovations that will reduce the cost and improve the efficiency of induction accelerators. In the author's opinion, the technical outlook for HIF is very good. 


\section{FIGURE CAPTIONS}

Figure 1. Block diagram of the standard multi-beam induction accelerator concept for heavy-ion fusion. The various accelerator systems and beam manipulations are shown. The values of kinetic energy and beam current are approximate, but most detailed accelerator designs have values close to those given.

Figure 2. A generic spherical target. Spherical targets require nearly uniform illumination produced by a large number of beams. Only one beam is shown.

Figure 3. A generic axial target. Such a target is suitable for one-sided or two-sided illumination. Only one beam is shown.

Figure 4. A simplified diagram of a ferromagnetic induction core. The pulsing circuit is conceptually represented by a capacitor and a switch. In practice a metal cavity surrounding the core confines the acceleration field to a gap in the beam line.

Figure 5. A schematic diagram of a magnetic quadrupole lens illustrating the scaling law that follows from Ampere's law. 


\section{REFERENCES}

[1] C.M. CELATA, A. FALTENS, W.M. FAWLEY, L.J. LASLETT, and I. HABER, "Simulation studies of space-charge-dominated beam transport in large aperture ratio quadrupoles," these proceedings.

[2] A.B. LANGDON and W.M. FAWLEY, "Charge neutralization of heavy-ion ICF driver beams, " these proceedings.

[3] D.D-M. HO, I. HABER, R. CRANDALL, and S.T. BRANDON, "Octupole correction of geometric aberration for high current heavy-ion fusion beams," Particle Accelerators 36, 141 160 (1991).

[4] E.P. LEE, "Longitudinal instability in heavy-ion-fusion induction linacs," these proceedings.

[5] J.J. BARNARD, F. DEADRICK, A. FRIEDMAN, D.P. GROTE, L.V. GRIFFITH, H.C. KIRBIE, V.K. NEIL, M.A. NEWTON, A.C. PAUL, W.M. SHARP, H.D. SHAY, R.O. BANGERTER, A. FALTENS, C.G. FONG, D.L. JUDD, E.P. LEE, L.L. REGINATO, S.S. YU, and T.F. GODLOVE, "Recirculating induction accelerators for heavy ion fusion," these proceedings.

[6] C.M. CELATA, R.O. BANGERTER, W.W. CHUPP, S. EYLON, A. FALTENS, W.M. FAWLEY, T.J. FESSENDEN, C.G. FONG, K. HAHN, E. HENESTROZA, D.L. JUDD, E.P. LEE, C. PETERS, L.L. REGINATO, P.A. SEIDL, S. YU, J.J. BARNARD, Y.-J. CHEN, A. FRIEDMAN, D.P. GROTE, D.W. HEWETT, and M.A. NEWTON, "The ILSE experimental program," these proceedings.

[7] S. EYLON, E. HENESTROZA, W.W. CHUPP, and S. YU, "Low emittance 0.8A K+ ion source for the LBL induction linac system experiment (ILSE)," these proceedings.

[8] K. HAHN, C. CELATA, A. FALTENS, D. JUDD, P. SEIDL, and E. LEE, "Transverse beam combiner for ILSE," these proceedings.

[9] L.L. REGINATO, "Longitudinal instability in heavy-ion-fusion induction linacs," these proceedings.

[10] T. FESSENDEN, R. BANGERTER, D. BERNERS, J. CHEW, S. EYLON, A. FALTENS, W. FAWLEY, C. FONG, M. FONG, K. HAHN, E. HENESTROZA, D. JUDD, E. LEE, C. LIONBERGER, S. MUKHERJEE, C. PETERS, C. PIKE, G.

RAYMOND, L. REGINATO, H. RUTKOWSKI, P. SEIDL, L. SMITH, D. VANECEK, S. YU, F. DEADRICK, A. FRIEDMAN, L. GRIFFITH, D. HEWETT, M. NEWTON, and H. SHAY, "ILSE: The next step toward a heavy ion induction accelerator for inertial fusion energy," Plasma Physics and Controlled Nuclear Fusion Research 1992, Interational Atomic Energy Agency Vienna, 1993, in press. 


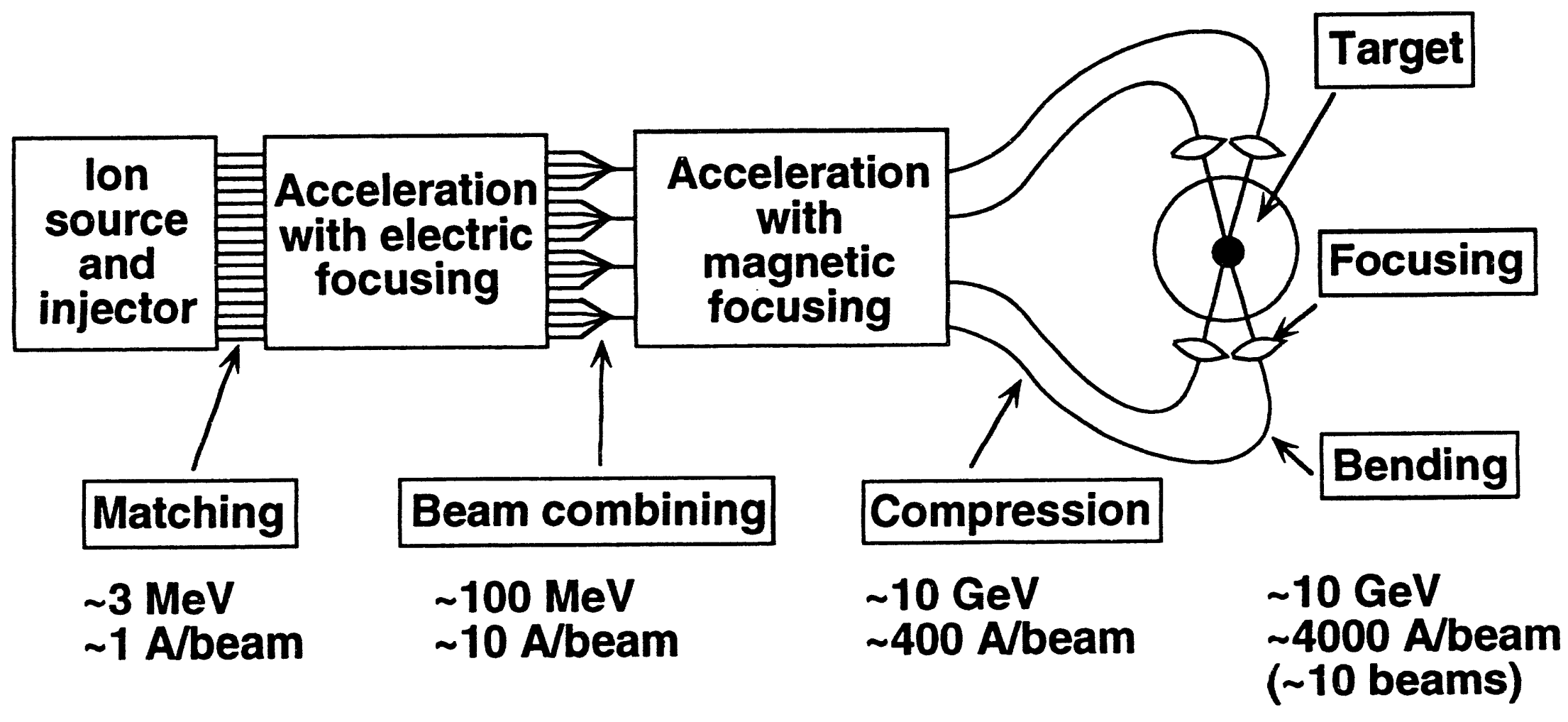




\section{Generic spherical target}

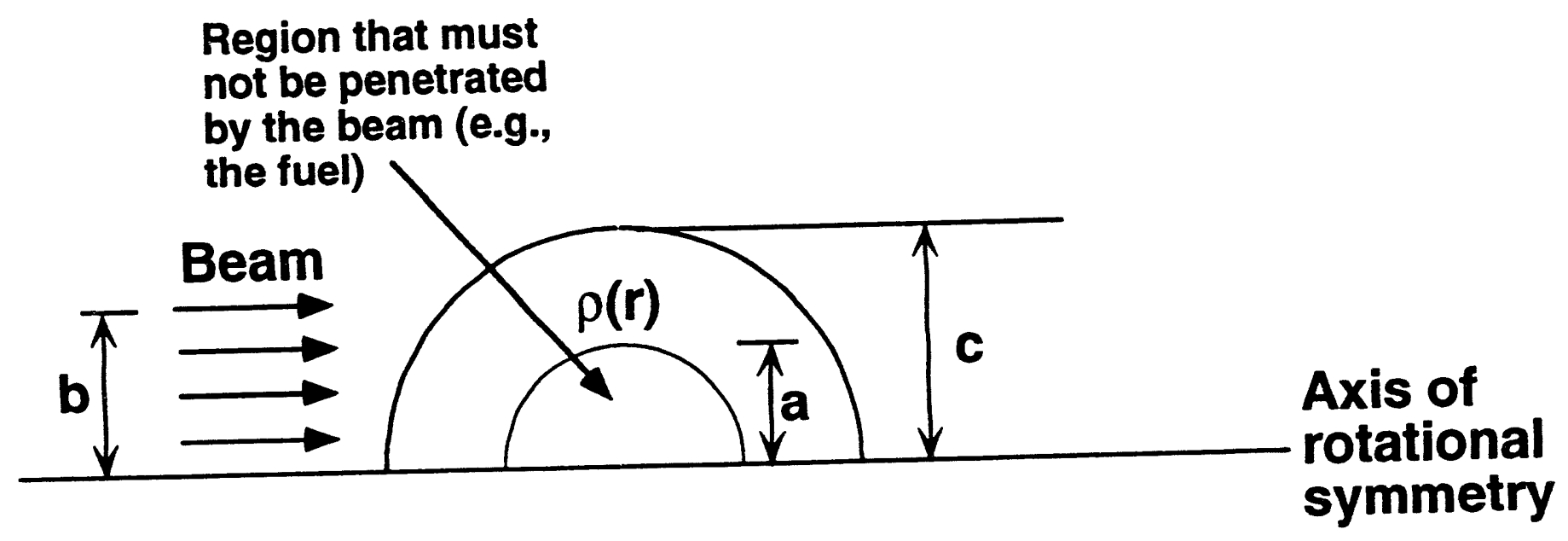

Generic axial target

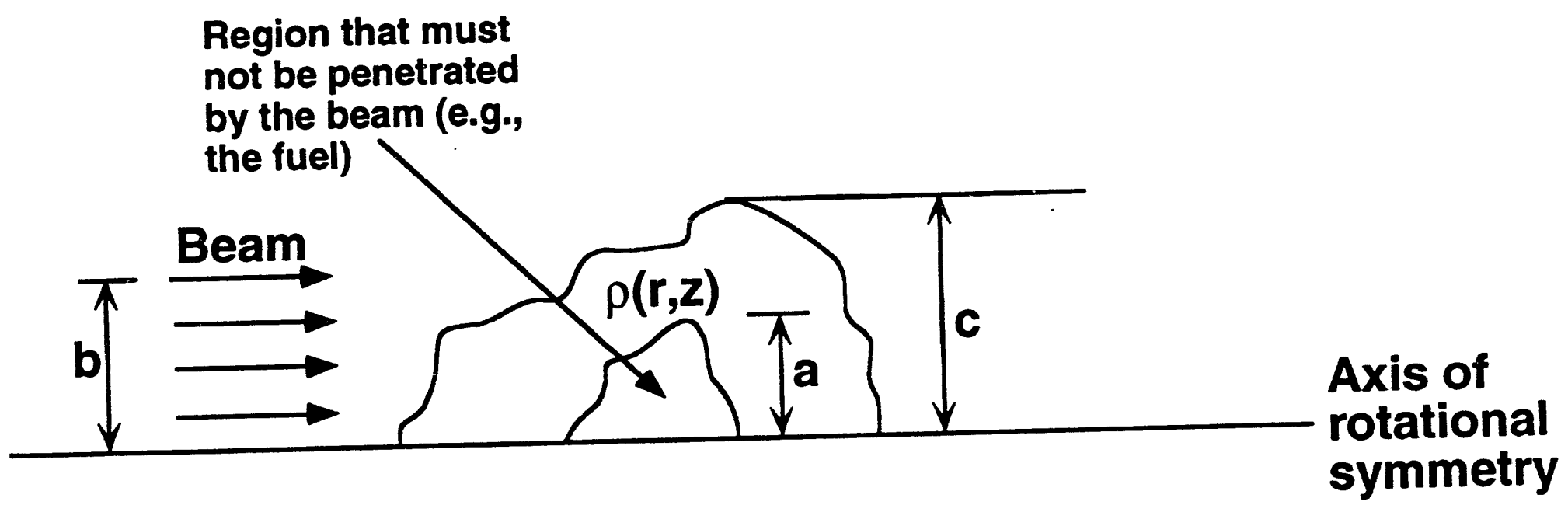




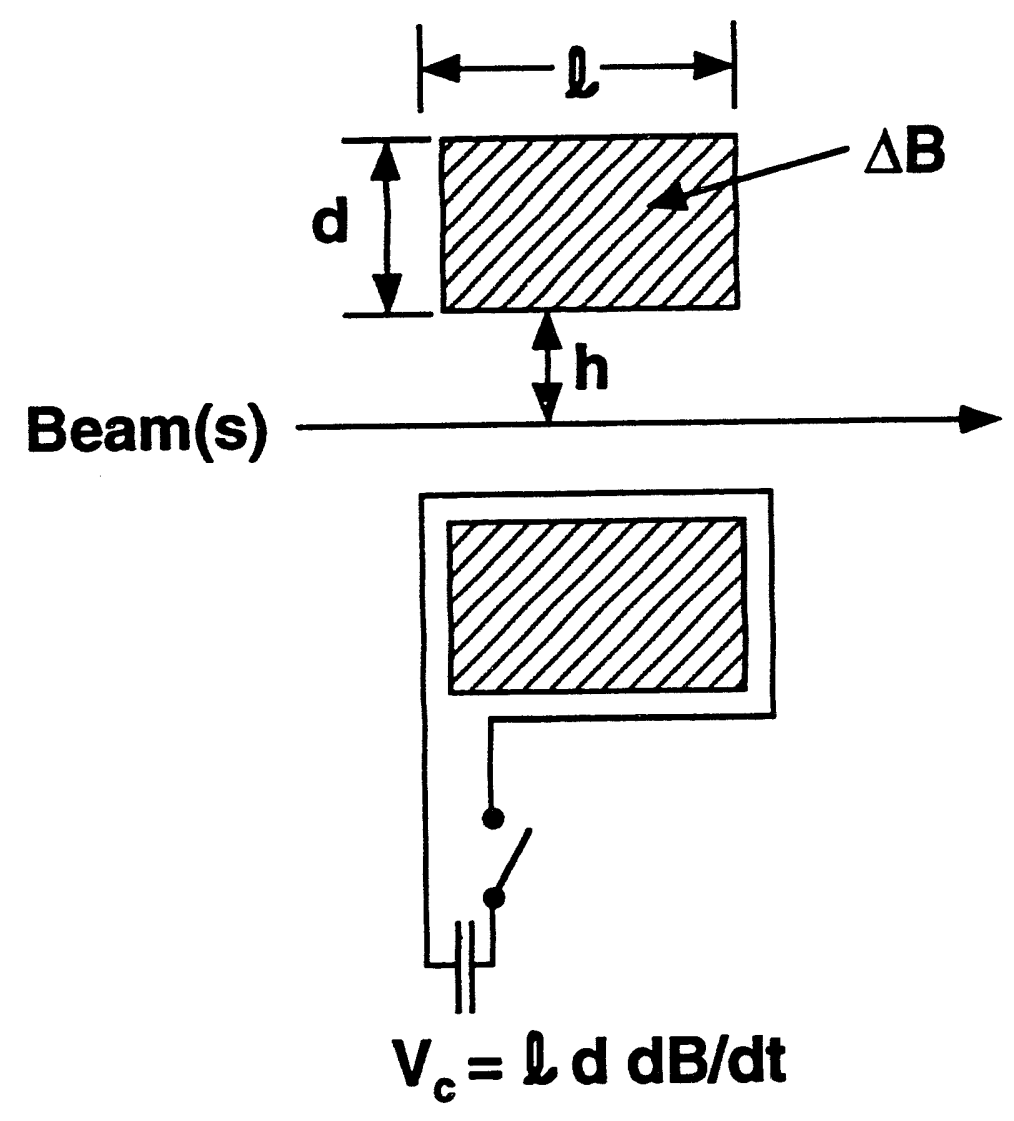




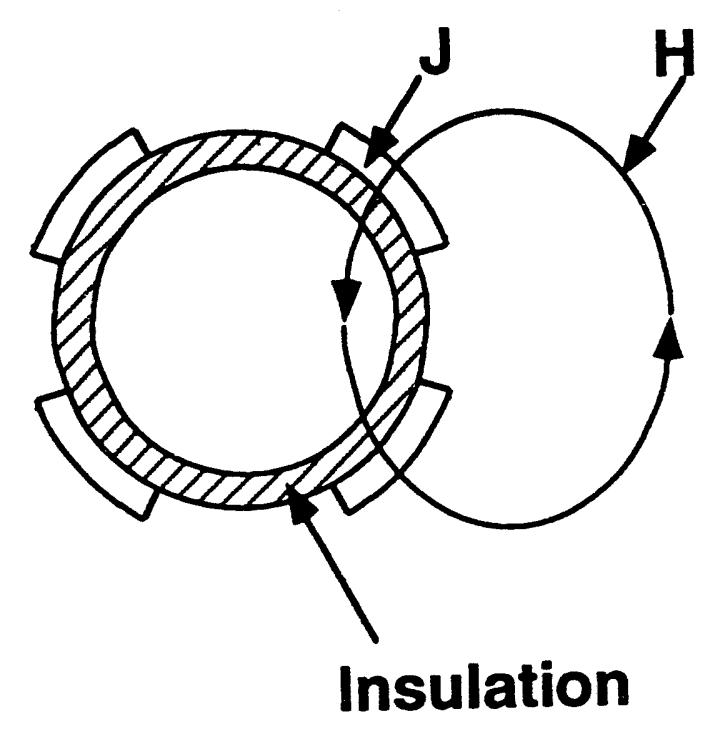




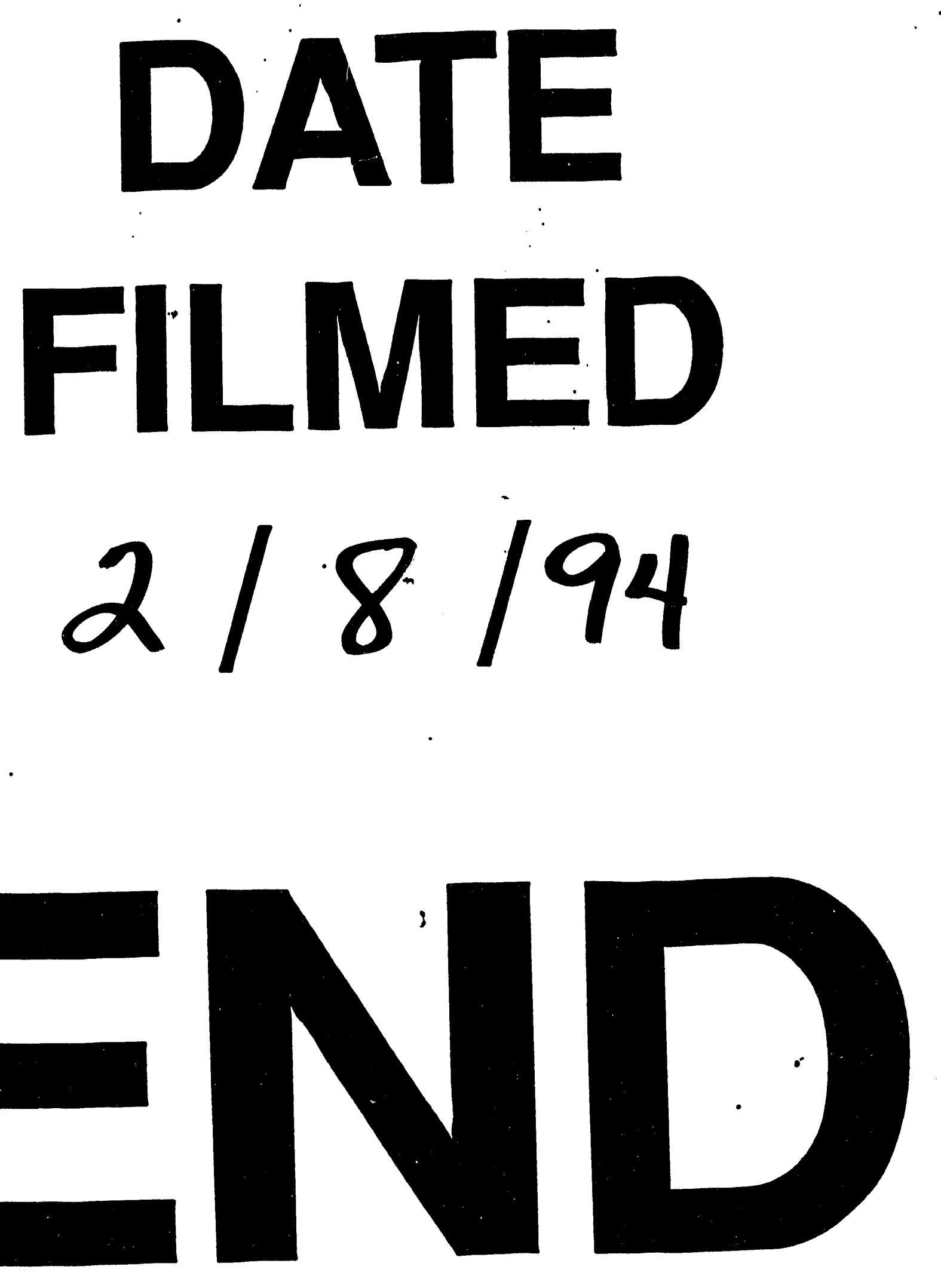


